\title{
Characterising (U)LIRGs using SALT/RSS
}

\author{
Rajin Ramphul*it \\ University of Cape Town, South Africa \\ South African Astronomical Observatory, South Africa \\ E-mail: rajin250@yahoo.com
}

\section{Petri Väisänen}

South African Astronomical Observatory, South Africa

Southern African Large Telescope (SALT), South Africa

E-mail: petriesaao.ac.za

\section{Kurt Van der Heyden}

University of Cape Town, South Africa

E-mail: heydeneast.uct.ac.za

\section{Abiy Getachew Tekola}

South African Astronomical Observatory, South Africa

E-mail: atekola@gmail.com

We present our ongoing work on a sample of 40+ Luminous Infrared Galaxies (LIRGS) which have been observed with SALT/RSS. We elaborate on the data acquisition and the data reduction pipeline that we devised to reduce the set efficiently. We explain how we intend to extract galaxy kinematics, stellar populations, star formation histories, gas ionization and metallicity from the data. Finally, we present some of the ongoing work on a few cases of galaxies in the sample.

SALT Science Conference 2015 -SSC2015-

1-5 June, 2015

Stellenbosch Institute of Advanced Study, South Africa

\footnotetext{
* Speaker.

${ }^{\dagger}$ I would like to acknowledge the UCT/SKA scholarship for the funding of my $\mathrm{PhD}$.
} 


\section{Introduction}

\subsection{LIRGs and ULIRGs}

LIRG is a designation for galaxies which have luminosities ranging $10^{11} L_{\odot} \leq L_{I R}<10^{12} L_{\odot}$, and their more energetic counterparts, the Ultra-LIRGs (ULIRGS), are classified as $10^{12} L_{\odot} \leq$ $L_{I R}<10^{13} L_{\odot}$. LIRGs and ULIRGS are powered by extreme star formation, AGN activity, or a combination of both processes. The emission of the mentioned processes are absorbed at shorter wavelength and re-emitted in the infrared we detect. Most LIRGs and ULIRGs are interacting or merging galaxy systems. Figure 1 gives a general overview of how their interaction stage, as well as how the underlying source of the luminosity, depend on their IR luminosity. While it is clear that AGN activity grows with luminosity, and more advanced merger systems are found at the higher luminosity bins, the process happening at the various stages and the cause-effect relations in the systems are still quite loosely constrained.
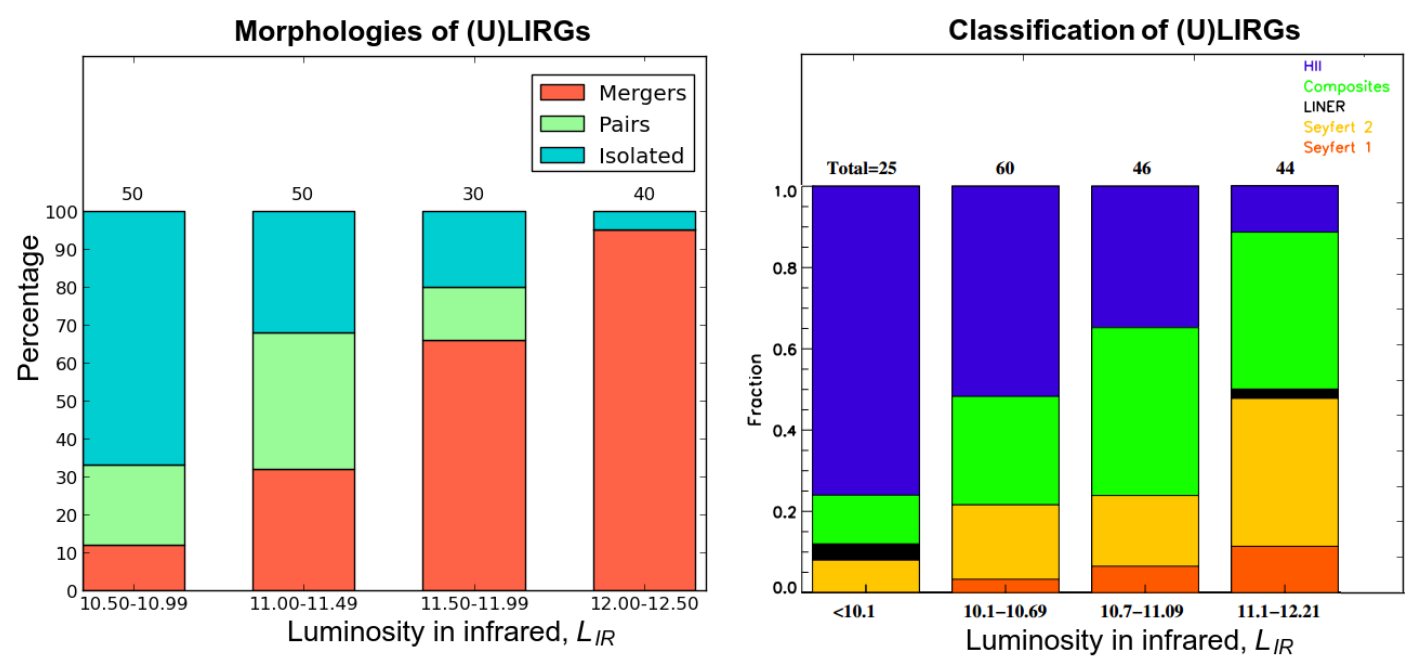

Figure 1: Right panel : The interaction stages of Infrared Galaxies versus IR luminosity. It clearly shows that IR luminosity is directly correlated to strong interaction or mergers [11]. Left Panel : The number of AGN-driven galaxies increases with higher IR luminosity [16]. The figure is adopted from [11] and [16]

LIRGs and ULIRGs thus provide a good laboratory to study the relationship between AGN and starburst activity, and they are good targets to test galaxy evolution models via major mergers [12].

\subsection{The Sample}

The data sample we are working on consists of 40+ LIRGs, from the SUperNovae and starBurst in the InfraRed (SUNBIRD) survey [14], that are mostly southern targets. We studied the Super Star Clusters (SSCs) of the same sample using Adaptive Optics photometry from VLT/NACO and partly from Gemini/ALTAIR/NIRI (for more in-depth information please see [9] and [15] . The galaxies are in the local universe at distances ranging approximately from 40 to $200 \mathrm{Mpc}$. They have been selected to have a diverse representation of interactions ranging from isolated cases to interacting systems and merging cases. For this work, we are specifically interested in the spectroscopic data obtained from SALT/RSS. 


\section{SALT Data}

The data was acquired over a span of 3 years, from late 2011 to 2014, covering different proposals. We chose to use two main grating settings for all the targets. A low resolution VPH grating, PG0900, with resolution of $R \sim 1000$ was used for all the targets. The rest frame wavelength coverage was $3200-6700 \AA$ and is therefore suited for stellar population modelling, metallicity and extinction measurements. The second setting that was selected made use of PG1800 - a medium resolution VPH grating at $R \sim 3000$. Galaxy kinematics and gas inflows will be modelled using this set-up which covers the rest frame region of $5600-6930 \AA$. More than 100 blocks of data were obtained for this program. The default Position Angle (PA) of the observation was usually aligned with the target's major axis. In cases where interesting star forming regions, or companion galaxies, were offset to the major axis, the galaxy was observed at another PA as well.

To be able to efficiently reduce this amount of data uniformly, a pipeline ${ }^{1}$ was written mainly in $\mathrm{IRAF}^{2}$ and Python. The flowchart of the data reduction pipeline is given in the Figure 2. The end result are $2 \mathrm{D}$ rectified frames that are wavelength calibrated, flux calibrated, and corrected for galactic extinction.

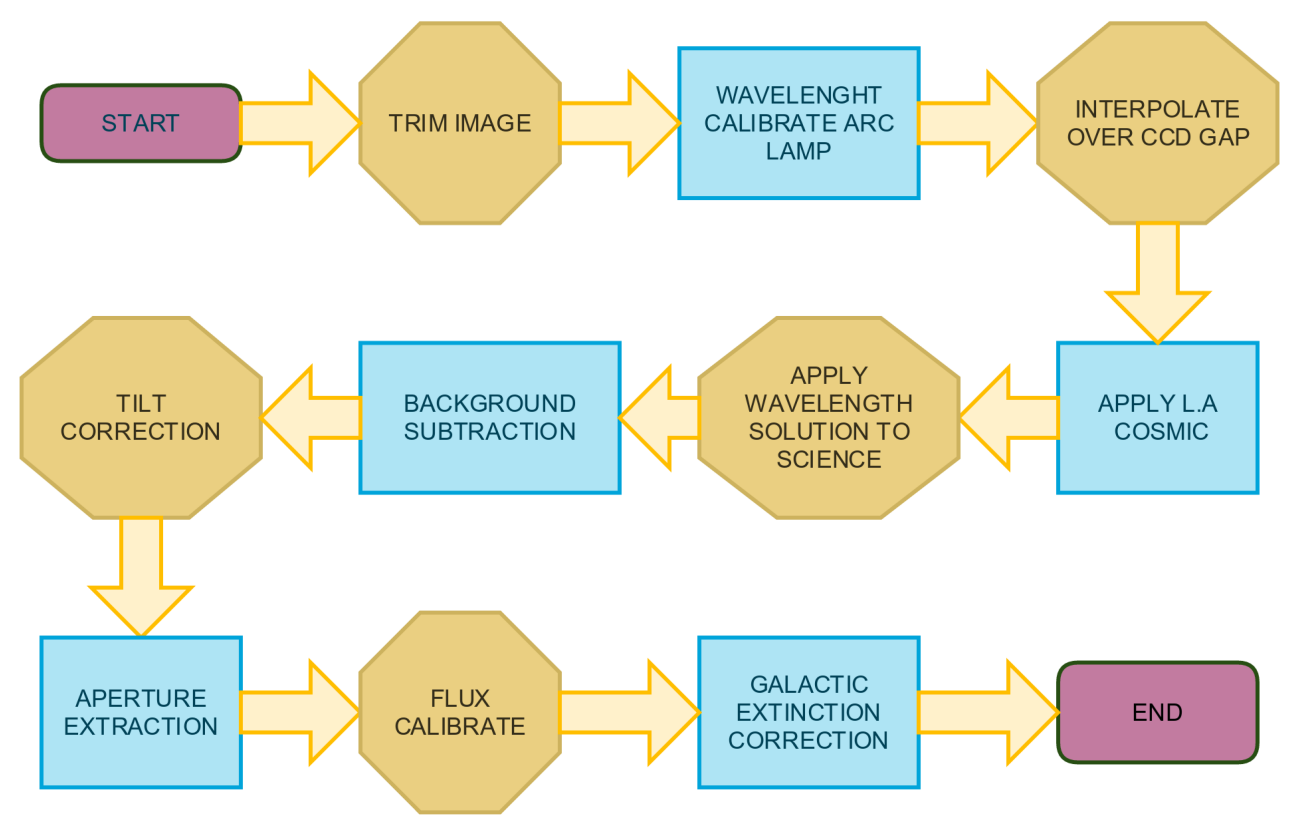

Figure 2: The spectral data reduction step-by-step flow

\section{Preliminary Results and Analysis}

\subsection{Stellar population modelling}

Modelling stellar populations (SP) of LIRGs is a challenge due to the complex star formation histories (SFH). One approach is to use photometry to probe the young population in clusters as was

\footnotetext{
${ }^{1}$ The pipeline can be downloaded here https : //github.com/rajin/rajin_code

${ }^{2}$ IRAF: the Image Reduction and Analysis Facility is distributed by the National Optical Astronomy Observatory.
} 
done for one galaxy in, e.g. [13]. In this work we focus on the SP modelling of LIRGs via spectral analysis which up to now is a relatively unexplored field; there have a been a handful of uniform studies of ULIRGs specifically, e.g. [4]. We chose the publicly available code STARLIGHT [2] which makes use of the inversion technique. The code is given the observed 1D spectrum and a basis which consists of several Single Stellar Populations (SSP) as input. The observed spectrum must be calibrated, extinction (Milky Way) corrected, at rest wavelength and with emission lines masked. The output from STARLIGHT is a population vector composed of a combination of SSPs, which makes up the star formation history of the object. To select the basis to feed to the code, we make use of the Diffusion map method [10]. This method provides a rigorous approach to sampling the age-metallicity parameter space evenly, hence decreasing degeneracies in results produced using this approach. Figure 3 shows an example of a starlight fit compared to the original spectrum. Figure 4 shows the SFH of the nuclear region of NGC6000, derived from the population vector.

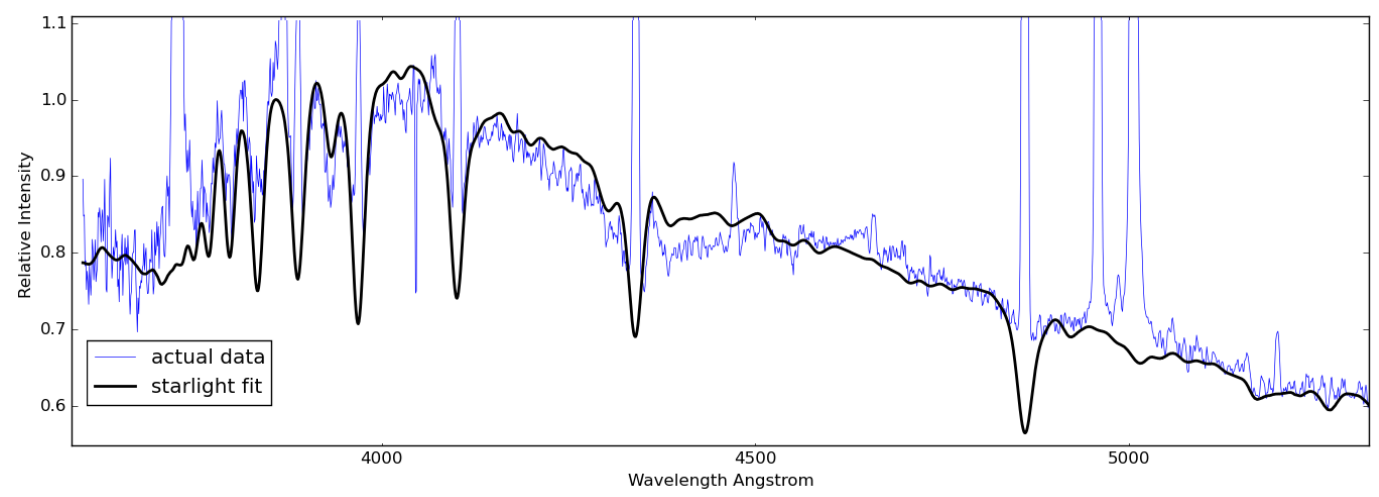

Figure 3: Starlight fit of ESO221-IG008 (Black Line) vs the actual data (Blue Line). Starlight fits are normalised at $\lambda_{o}=4020 \AA$

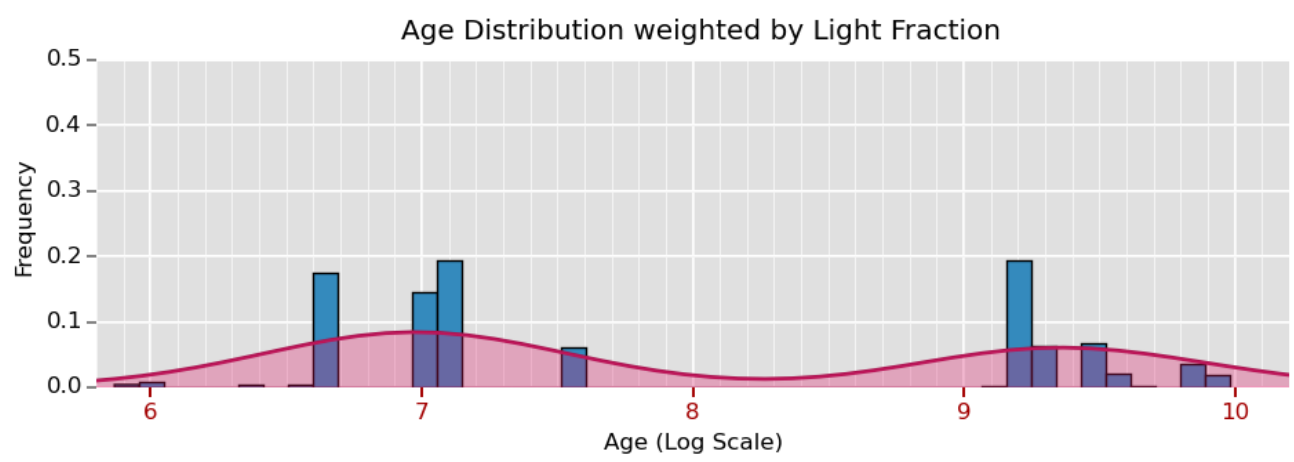

Figure 4: The light fraction distribution as a function of age for NGC6000. The red line is a smoothing kernel which represents the star formation history of the galaxy. We observe a SFH with essentially two peaks, dominated by the presence of a young approximately $10 \mathrm{Myr}$ old population (this is the starburst responsible for the LIRG classification of this target) and an old population formed a few Gyr ago. 


\subsection{Gas Ionisation}

After applying STARLIGHT, we can measure emission lines by subtracting the stellar contribution of light (given by STARLIGHT) from the total galaxy light (observed spectrum). The continuum subtracted spectra, which now only contains gas emission, are then fed to a python code measuring fluxes of emission lines by Gaussian fits. We use the well known BPT diagram [1] which differentiates star-forming galaxies from AGN driven galaxies. Figure 5 shows the BPT diagram for a sub sample of galaxies whose nuclear region were fit by STARLIGHT. From the plot it can be seen that this sub-sample of galaxies is mostly star forming dominated.

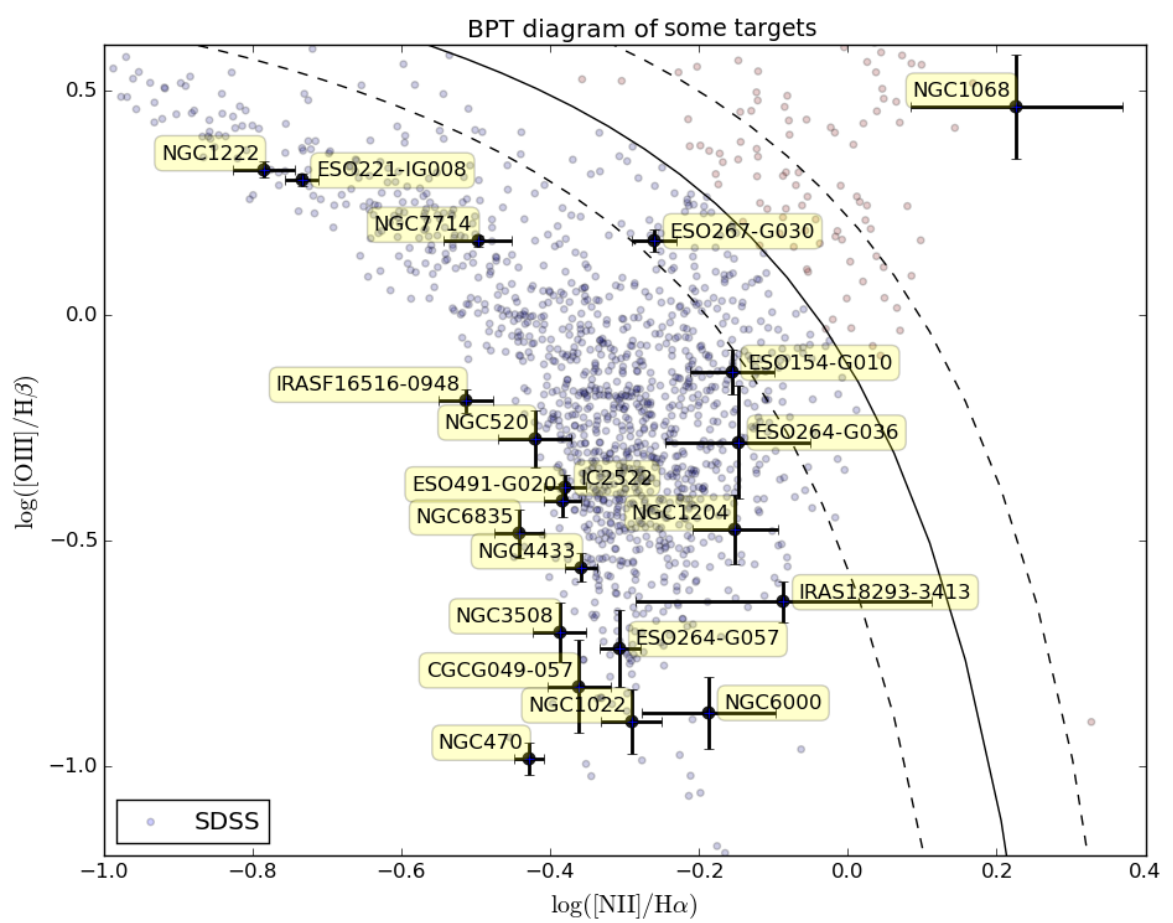

Figure 5: Diagnostic diagrams of emission line ratios of $[\mathrm{NII}] / \mathrm{H} \alpha$ against $[\mathrm{OIII}] / \mathrm{H} \beta$. The background of the plot consists of points from the SDSS sample which are shown for comparison purposes. The solid curve is that from [6], representing the theoretical separation between HII dominated region (below the curve) to that dominated by shock excitation (above the curve).

\subsection{Metallicity}

We use the Gaussian fitting method to extract the fluxes of all the emission lines from our continuum-subtracted spectrum. The intrinsic fluxes are then recovered by correcting for the internal extinction (see [5] and [7]). The intrinsic line ratio, their corresponding equivalent width (EW), the absorption coefficient, $\mathrm{C}(\mathrm{H} \beta)$ and the electron temperature $T_{e}(\mathrm{OIII})$ are solved through an iterative process and uncertainties are calculated via Monte-Carlo method. The oxygen abundances are then calculated with 3 different methods. The $\mathrm{N} 2=[\mathrm{NII}] / \mathrm{H} \alpha$ index and $\mathrm{O} 3 \mathrm{~N} 2=\frac{[O I I I]}{H \beta} / \frac{[N I I]}{H \alpha}$ are empirical methods from [8]. The third index used is the DO2 uses value from [NII]/[OII] and $[\mathrm{NII}] / \mathrm{H} \alpha$ to calculate metallicity [3]. Figure 6 shows an example of the spatially resolved metallicity determination for ESO 221-008. In this case there is no clear gradient evident, as is expected 
for galaxies where interactions have triggered inflows of less enriched gas from the outskirts thus evening out any differences. The abundance gradients in our SUNBIRD sample will be correlated carefully with the morphological details and kinematics of the target galaxies.

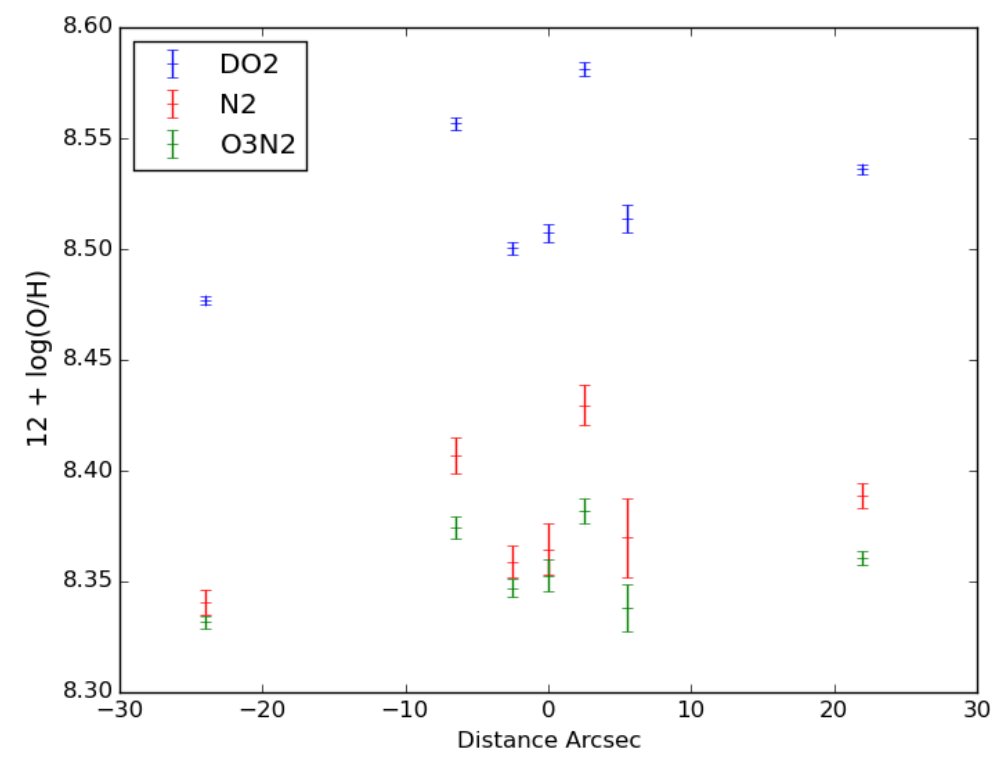

Figure 6: Metallicity as a function of radius for ESO221-IG008 found at a distance of $45.9 \mathrm{Mpc}$ (each arcsecond on sky represents $222 \mathrm{pc}$ in radial direction). The error bars shown are from propagated uncertainties while each method has its own intrinsic systematic errors which are not shown here. By extracting apertures around the nucleus, we are able to derive metallicity profiles for the targets (here the profile is flat).

\section{Concluding remarks}

The work presented is a flavour of what we want to achieve with the SUNBIRD survey. We intend to model the SSPs, determine the line ratios and metallicities for the entire sample of galaxy. We are also in the process of getting the masses of galaxies via the modelling of the kinematics (not discussed here). All these will be analysed as a function of radial distance from the the nuclei and as a function of the interaction stage and environment of the galaxies.

\section{References}

[1] Baldwin, J. A., Phillips, M. M., \& Terlevich, R., 1981, PASP, 93, 5B

[2] Cid Fernandes, R., Mateus, A., Sodré, L., Stasińska, G., \& Gomes, J. M., 2005, MNRAS, 358, 363

[3] Denicoló, G., Terlevich, R., \& Terlevich, E., 2002, MNRAS, 330, 69D

[4] Hou, L.G., et al., 2011, ApJ, 732, 72

[5] Izotov, Y. I., Thuan, T. X., \& Lipovetsky, V. A., 1994, ApJ, 435, 647I

[6] Kewley, L. J., Dopita, M. A., Sutherland, R. S., Heisler, C. A., \& Trevena, J., 2001, ApJ, 556, 121K

[7] Kniazev, A. Y., Zijlstra, A. A., Grebel, E. K., et al., 2008, MNRAS 388, 1667K 
[8] Pettini, M., \& Pagel, B. E. J., 2004, MNRAS, 348L, 59P

[9] Randriamanakoto, Z., et al. 2013, ApJ, 577, L38

[10] Richards, J. W., Freeman, P. E., Lee, A. B., \& Schafer, C. M., 2009, MNRAS ,399 ,1044R

[11] Sanders, D. B, Mirabel, I. F.,1996, A\&A, 34, 749S

[12] Springel, V., 2005, MNRAS, 364, 1105S

[13] Vaisanen, P., Barway, S., \& Randriamanakoto, Z., 2014, ApJ, 797, L16

[14] Väisänen, P., Randriamanakoto, Z., Escala, A., 2014, Massive Young Star Clusters Near and Far: From the Milky Way to Reionization, 185

[15] Väisänen, P., 2015, Current proceedings

[16] Yuan, T.-T., Kewley, L. J., \& Sanders, D. B. 2010, Apj, 709, 884 